\title{
Results of Recent Trials on the Progression of Coronary Artery Disease and Recurrent Ischemic Events: Implications for Interventional Cardiology
}

\author{
BERTRAM PITT, M.D. \\ From the Division of Cardiology, Department of Internal Medicine, University of Michigan Medical Center, Ann Arbor, Michigan
}

\section{Introduction}

There is increasing evidence to suggest that secondary prevention of ischemic heart disease by low density lipoprotein-cholesterol (LDL-C) reduction as a result of administration of an HMG-CoA reductase inhibitor, or dietary modification with alpha-linolenic acid is effective in reducing recurrent nonfatal and fatal myocardial infarction. ${ }^{1-4}$ The debate ${ }^{5}$ concerning lipid reduction or dietary modification in primary prevention in regard to a potential offset in the reduction of cardiovascular mortality by an increase in noncardiovascular mortality due to accidents, suicide, and/or cancer does not seem relevant in the setting of secondary prevention where the accumulating evidence of a marked reduction in recurrent ischemic events ${ }^{1}$ does not appear likely to be offset by an excess in noncardiovascular mortality, even if it should exist.

Data from the PLAC-I study, ${ }^{3}$ an angiographic study of over 400 patients with significant coronary artery disease randomized to the HMG-CoA reductase inhibitor pravastatin 40-mg daily or placebo in patients maintained on an NCEP-step 1 lipid reduction diet shows a significant reduction in LDL-C and the progression of coronary artery disease in those patients randomized to pravastatin. Of interest in this study, as in the CCAIT study ${ }^{6}$ using the HMG-CoA reductase inhibitor lovastatin, is the finding that the major effect of pravastatin and lovastatin was on progression of minimal coronary arterial lesions and new lesion for-

Address for reprints: Bertram Pitt, M.D., Division of Cardiology, Department of Internal Medicine, University of Michigan Medical Center, Ann Arbor, MI 48109. Fax: (313) 936-7641.

Submitted for publication July 22, 1994; accepted for publication July $25,1994$. mation. The reduction in the progression of minimal coronary arterial lesions progression and new lesions formation was associated with a significant reduction in recurrent myocardial infarction in the PLAC-I study ${ }^{3}$ and a trend toward a reduction in recurrent ischemic events in the CCAIT study. ${ }^{6}$ The importance of these findings of an association between a reduction in the progression of minimal coronary arterial lesions and new lesion formation with recurrent myocardial infarction can be seen from previous serial angiographic studies, which have pointed out that a large percentage of recurrent myocardial infarcts arise from minimal coronary artery lesions and/or new lesion formation. ${ }^{7-10}$ Little et al. ${ }^{7}$ were the first to emphasize this association. In a serial angiographic study they noted that a large percentage of myocardial infarctions arose from minimal coronary lesions on the baseline coronary arteriogram obtained many months or years prior to the infarction. The importance of minimal coronary artery lesion progression and/or new lesion formation in the pathogenesis of recurrent myocardial infarction can also be seen in the recent study of Ogawa et al. ${ }^{10}$ These authors studied a group of patients with myocardial infarction and single vessel coronary arterial disease. Over a mean follow-up period of 110 months they noted 19 recurrent episodes of myocardial infarction. Eighteen of these 19 episodes occurred at sites different from the index infarction, suggesting the importance of minimal coronary artery lesions and/ or new lesion formation in the pathogenesis of recurrent infarction. The relatively rapid prevention of recurrent myocardial infarction by LDL-C reduction in studies with pravastatin including the Pravastatin Multinational Study, ${ }^{11}$ PLAC II, ${ }^{12}$ and PLAC I ${ }^{3}$ has been attributed to reversal of endothelial dysfunction, pre- 
vention of plaque rupture. and/or prevention of thrombosis after plaque rupture. ${ }^{13}$

Endothelial dysfunction has been seen in angiographically normal coronary arteries in patients with adjacent coronary arterial lesions and in patients with multiple coronary artery risk factors without any evidence of angiographically significant coronary arterial lesions. ${ }^{14,15}$ Recent studies have shown that this endothelial dysfunction can be reversed by LDL-C reduction by cholestyramine ${ }^{16}$ or the HMG-CoA reductase inhibitors lovastatin ${ }^{17}$ and pravastatin ${ }^{18}$ within a period of 6 months. LDL-C reduction by dietary and lipid lowering therapy has also been shown to improve coronary arterial flow reserve as assessed by positron emission tomography. ${ }^{19}$ The improvement in coronary arterial flow reserve within a period of 6 months of LDL-C reduction is associated with a reduction in the incidence of symptomatic angina pectoris. ${ }^{19}$

Davies and Thomas ${ }^{20}$ and others ${ }^{21.22}$ have described the factors predisposing to plaque rupture. It is likely that a reduction of cholesterol in the lipid rich core of the early atherosclerotic lesion would minimize the likelihood of plaque rupture. More intriguing and perhaps the best explanation for the relatively rapid reduction in ischemic events seen in the pravastatin multinational trial, ${ }^{11}$ PLAC-II, ${ }^{12}$ and most recently PLAC-I ${ }^{3}$ is a direct or indirect effect on platelet induced thrombosis. Pravastatin has been shown to inhibit plateletinduced thrombosis in an ex vivo vascular model. ${ }^{23} \mathrm{~A}$ reduction in platelet-induced thrombosis either directly or indirectly through reversal of endothelial dysfunction could account for a relatively rapid reduction in recurrent ischemic events. Further evidence linking inhibition of platelet-induced thrombosis to a relatively early reduction in ischemic events comes from the recent LYON Diet Heart Study. ${ }^{4}$ In this study 605 patients after a first myocardial infarction were randomized to a control group or to an experimental diet group in which patients received a diet rich in alphalinolenic acid. Alpha-linolenic acid is a precursor of the N-3 long chain fatty acids and has been shown to have a beneficial effect on platelet reactivity. ${ }^{24} \mathrm{~Pa}$ tients assigned to the experimental diet group had a close to $70 \%$ reduction in recurrent coronary events and cardiac deaths over a follow-up period of 5 years. This reduction in recurrent ischemic events and cardiac death was seen without a signifcant reduction in LDL-C or HDL-C between the control and experimental groups. The reduction in recurrent ischemic events was noted relatively early as evidenced by a diver- gence in the survival and recurrent event curves after several months, rather than the several years noted in the POSCH trial with intestinal bypass ${ }^{25}$ or cholestyramine. ${ }^{26}$

Despite the accumulating evidence for the effectiveness of secondary prevention of recurrent ischemic events either by LDL-C reducing agents, ${ }^{1,4}$ or dietary interventions, such as alpha-linolenic acid ${ }^{4}$ many cardiologists have been reluctant to incorporate this information into their practices. For example, in a recent study only $25 \%$ of patients undergoing percutaneous transluminal coronary angioplasty (PTCA) had a determination of their serum lipids during a 2-year follow-up and even a smaller percentage received any lipid lowering drugs or effective dietary therapy. ${ }^{27}$

What are the implications of these recent studies for interventional cardiology? First, we should be more vigorous in evaluating and correcting coronary artery risk factors in patients undergoing PTCA and other interventions. The realization that a large percentage of recurrent ischemic events arise from progression of minimal coronary arterial lesions or new lesion formation suggests that if we are to be successful in preventing recurrent myocardial infarction and death in our patients that we need to do more than alleviate hemodynamically significant lesions by PTCA or other devices. PTCA and restoration of coronary arterial flow reserve are important in relieving patient symptoms and improving exercise performance, ${ }^{28}$ but cannot be expected to be optimal and have not been shown to be effective in preventing recurrent myocardial infarction or death since many of these recurrent ischemic events occur due to plaque rupture and thrombosis of minimal or new coronary artery lesions. Relief of symptoms, improved exercise performance, and life style are certainly worthy goals and a clear indication for PTCA and other interventions. Focusing only on relief of these hemodynamically and angiographically significant lesions is, however, not adequate given the accumulating evidence that we have the means to relatively rapidly and effectively reduce recurrent ischemic events. ${ }^{3.4}$

The second implication of these recent studies is more far reaching and controversial. If as suggested above, many recurrent myocardial infarctions are a result of plaque rupture and thrombosis of minimal or new coronary artery lesions ${ }^{7-10}$ it is unlikely that PTCA of a single coronary artery vessel and likely multiple vessels will be shown to have a significant effect on a reduction in recurrent myocardial infarc- 


\section{PROGRESSION OF CORONARY ARTERY DISEASE}

tion. PTCA of a single coronary artery should be viewed as effective symptomatic therapy. All too often, however, PTCA, especially postinfarction, is performed on the basis of angiographic findings in asymptomatic or mildly asymptomatic individuals. Without clear data from prospective well-controlled trials demonstrating a beneficial effect of PTCA in reducing recurrent infarction and death in this situation it is hard to understand how we can continue to justify this procedure under these circumstances if we are to claim in this rapidly changing health care environment that we wish to provide our patients the best and most cost-effective therapy. The recent trials described above have important implications even for symptomatic patients. The evidence of a relatively rapid reduction in symptomatic angina pectoris in the study by Gould and colleagues ${ }^{19}$ and the rapid reduction in recurrent ischemic events with pravastatin, 3,11,12 and even more impressively by dietary modification with alpha-linolenic acid ${ }^{4}$ suggests that it might be reasonable, at least in those with stable angina pectoris and moderately preserved exercise performance to attempt effective dietary modification and/or LDL-C reduction for a period of 6 months before resorting to PTCA. If at the end of a 6-month trial period, the time period in which it has been shown that endothelial dysfunction is reversed by LDL-C reduction ${ }^{16-18}$ and symptoms relieved, ${ }^{19}$ the patient is still symptomatic despite optimal medical therapy including nitrates, and beta adrenergic blocking agents as well as attempts at dietary modification such as with the Lyon Heart Diet ${ }^{4}$ or LDL-C reduction with an HMG-CoA reductase inhibitor, ${ }^{3.11 .12}$ PTCA or other interventional procedures should be attempted for symptomatic relief.

The above discussion is not meant to denigrate the importance of PTCA and other interventions in correcting high grade coronary arterial lesions and thereby, relieving symptoms and likely reducing ischemic events. Clearly, many recurrent ischemic events are associated with high grade coronary arterial lesions. ${ }^{7}$ A strategy that focuses only on these lesions or provides relatively expensive and often incomplete symptomatic relief in those with only mild symptoms and/or moderately hemodynamically significant lesions is unlikely to pass scrutiny in the new era of cost containment nor should it even in the current era.

\section{References}

1. Law MR, Wald NJ. Thompson NJ. By how much and how quickly does reduction in serum cholesterol concentration lower risk of ischemic heart disease? $\mathrm{Br}$ Med J 1994;308: $367-372$.

2. Blackburn DH, Hodis HN. Arterial imaging and atherosclerosis reveral. Arterioscler Thromb 1994;14:177-192.

3. Pitt B, Mancini GBJ, Ellis SG, et al. for the PLAC I Investigators. Pravastatin limitation of atherosclerosis in the coronary arteries (PLAC I). JACC 1994;1 A-484A, 131A.

4. deLorgeril M, Renaud S, Mamelle N, et al. Mediterranean alpha-linolenic acid-rich diet in secondary prevention of coronary heart disease. Lancet 1994;343:1454-1459.

5. Wittels EH, Hay JW, Gotto AM. Medical costs of coronary artery disease in the United States. Am J Cardiol 1990;65: $432-440$

6. Waters D, Higginson L, Gladsone P, et al for the CCAIT Study Group. Effects of monotherapy with an HMG-CoA reductase inhibitor on the progression of coronary atherosclerosis as assessed by serial quantitative arteriography. The Canadian Coronary Atherosclerosis Intervention Trial. Circulation 1994;89:959-968.

7. Little WC, Constantinescu M, Applegate RJ, et al. Can coronary angiography predict the site of a subsequent myocardial infarction in patients with mild-to-moderate coronary artery disease? Circulation 1988;78:1157-1166.

8. Nobuyoshi $M$, Tanaka $M$, Nosoka $H$, et al. Progression of coronary atherosclerosis: Is coronary spasm related to progression? JACC 1991;18:904-910.

9. Bruschke AVG, Kramer JR Jr, Bal ET, et al. The dynamics of progression of coronary atherosclerosis studies in 168 medically treated patients who underwent coronary arteriography three times. Am Heart J 1989;117:296-305.

10. Ogawa $\mathrm{H}$, Kawana M, Tamura K, e al. Long-term prognosis of medically treated patients with acute myocardial infarction and one-vessel coronary artery disease. Am J Cardiol 1994; 73:158-163.

11. The Pravastatin Multinational Study Group for Cardiac Risk Patients. Effects of pravastatin in patients with serum total cholesterol levels from 5.2 to $7.8 \mathrm{mmol} / \mathrm{iter}(200$ to $300 \mathrm{mg} /$ dL) plus two additional atherosclerotic risk factors. Am J Cardiol 1993;72:1031-1037.

12. Furberg CD, Byington RP, Crouse JR, et al. Pravastatin, lipids, and major coronary events. Am J Cardiol 1994;73:1133-1134.

13. Werns SW, Walton JA, Hsia $\mathbf{H H}$, et al. Evidence of endothelial dysfunction in angiographically normal coronary arteries of patients with coronary artery disease. Circulation 1989;79: 287-291.

14. Pearson TA, Marx HJ. The rapid reduction in cardiac events with lipid-lowering therapy: Mechanisms and implications. Am J Cardiol 1993:72:1072-1073.

15. Zeiher Am, Drexler H, Saurbier B, et al. Effects of age, atherosclerosis, hypercholesterolemia, and hypertension: Endothelium-mediated coronary blood flow modulation in humans. J Clin Invest 1993;92:652-662.

16. Leung WH, Lau CP, Wong CK. Beneficial effects of cholesterol-lowering therapy on coronary endothelium-dependent relaxation in hypercholesterolemic patients. Lancet 1993:341: 1496-1500.

17. Treasure CB, Talley JD, Stillabower ME, et al. and the Lovastatin Restenosis Trial Study group. Coronary endothelial responses are improved with aggressive lipid lowering therapy in patients with coronary atherosclerosis. Circulation 1993;88: I-368.

18. Egashira K, Hirooka $\mathrm{Y}$, Kai H, et al. Reduction in serum cholesterol with pravastatin improves endothelium-dependent coronary vasomotion in patients with hypercholesterolemia. Circulation 1994;89:2519-2524.

19. Gould KL, Martucci JP, Goldberg DI, et al. Short-term choles- 
terol lowering decreases size and severity of perfusion abnormalities by positron emission tomography after dipyridamole in patients with coronary artery disease. A potential noninvasive marker of healing coronary endothelium. Circulation 1994;89:1530-1538.

20. Davies MJ, Thomas AC. Plaque fissuring - the cause of acute myocardial infarction, sudden ischaemic death, and crescendo angina. Br Heart J 1985:53:363-373.

21. Fuster $\mathrm{V}$, Badimon L, Badimon $\mathrm{JJ}$, et al. The pathogenesis of coronary artery disease and the acute coronary syndromes (1). $N$ Engl J Med 1992;326:242-250.

22. Fuster V, Badimon L, Badimon JJ. et al. The pathogenesis of coronary artery disease and the acute coronary syndromes (2). N Engl J Med 1992;326:310-318.

23. Lacoste LL. Lam JYT. Hung J, et al. Pravachol decreases platelet thrombus formation in hypercholesterolemic coronary patients in conjunction with improvements in serum lipids. JACC 1994:1 A-484A:131 A
24. Renaud S, Nordoy A. Small is beautiful: Alpha-linolenic acid and eicosapentaenoic acid in man. Lancet 1983;1:1169.

25. Buchwald H, Varco RL, Matts JP, et al. Effect of partial ileal bypass surgery on mortality and morbidity from coronary heart disease in patients with hypercholesterolemia. Report of the Program on the Surgical Control of the Hyperlipidemias (POSCH). N Engl J Med 1990;323:946-955.

26. Lipid Research Clinics Program. The Lipid Research Clinics Coronary Primary Prevention Trial Results. JAMA 1984;251: $351-364$.

27. Silver $\mathrm{KH}$, Weiner BH, Borbone $\mathrm{ML}$, et al. Serum cholesterol levels after cronary angioplasty in men and women. JACC 1994: 1A-484A,299A.

28. Parisi AF, Folland ED, Hartigan P. A comparison of angioplaty with medical therapy in the treatment of single-vessel coronary artery disease: Veterans Affairs ACME Investigators. N Engl J Med 1992;326:10-16. 